\title{
A pilot study of an Enhanced Mental Health Assessment during routine and discharge medicals in the British Armed Forces
}

'Department of General Practice, Royal Centre for Defence Medicine, Birmingham, UK ${ }^{2}$ Academic Centre for Defence Mental Health, Weston Education Centre, London, UK ${ }^{3}$ Department of Community Mental Health, HM Naval Base, Portsmouth, UK

\section{Correspondence to} Capt M Aguirre, Royal Centre for Defence Medicine, ICT Research Park, Birmingham, Vincent Drive, B15 2SO, UK m.aguirre@doctors.org.uk

Received 7 March 2013 Accepted 9 March 2013 Published Online First 11 June 2013
To cite: Aguirre $\mathrm{M}$, Greenberg N, Sharpley J, et al. J R Army Med Corps 2014;160:27-31.

\author{
Marcela Aguirre, ${ }^{1}$ N Greenberg, ${ }^{2}$ J Sharpley, ${ }^{3}$ R Simpson, ${ }^{1}$ C Wall ${ }^{1}$
}

\begin{abstract}
Objectives A pilot study to assess the practicality of introducing an enhanced mental health assessment (EMHA) into all routine and discharge medicals of the UK Armed Forces in order to facilitate treatment prior to and on return to civilian life.
\end{abstract}

Methods A pilot study was conducted using an EMHA questionnaire with questions about depression, anxiety, post-traumatic stress disorder, alcohol use, sleep and anger/irritability. At pilot sites, the EMHA was completed during all routine and discharge medicals between May 2011 and July 2011. At the end of the study period, qualitative data were collected from participating medical officers and practice managers regarding their opinions about the pilot study.

Results The quantitative data revealed an average pickup rate for mental health $(\mathrm{MH})$ problems. Out of the four military medical centres who participated and the 325 questionnaires collected, one referral to a Department of Community Mental Health was made. $26(8 \%)$ patients were categorised as 'some concern and patient offered advice and/or reassurance'. The vast majority of patients were found to have no evidence of $\mathrm{MH}$ problems. However, using a validated alcohol screening tool, 64\% of service personnel were found to have a score indicating 'higher risk drinking'. Analysis of the qualitative data suggests that the EMHA is an easy tool to implement with minimal additional time and resources needed. The interviewees pointed out a number of limitations and suggestions for possible further studies.

Conclusions The pilot study successfully demonstrated that the EMHA questionnaire is easy to administer, does not take up a large amount of additional resources or manpower and provides a useful check of MH status. The study picked up an average number of $\mathrm{MH}$ cases and the questions on alcohol consumption highlighted that military personnel may be at a 'higher risk of drinking'.

\section{INTRODUCTION}

Service in the Armed Forces is generally associated with good mental and physical health. ${ }^{1}$ However, in the past few years, the mental health $(\mathrm{MH})$ of service personnel and veterans has become a topic of considerable concern for the Ministry of Defence, the National Health Service and society as a whole. ${ }^{2}$ The evidence collected on the health of UK Armed Forces personnel suggests that the majority of personnel do well both in service and after they return to civilian life. ${ }^{3}{ }^{4}$ However, a small proportion of personnel do suffer from formal MH disorders. Mental illness is a cause of morbidity and mortality and is associated with both homelessness and involvement in the Criminal

\section{Key messages}

- The prevalence of mental health illness in the Armed Forces is largely similar to that found in the UK civilian population, but operational deployment is a known risk factor for some mental health conditions.

- Stigma surrounding mental health is significant and may be preventing patients from seeking treatment.

- Routine and discharge medicals should address mental health as part of a full systems enquiry.

- Excessive alcohol consumption in the Armed Forces may be an issue.

- The Enhanced Mental Health Assessment is a useful and easily implemented tool, with minimal uplift in administrative resources and time. Incorporating this tool into routine and discharge medicals could assist in performing a more thorough mental health assessment.

Justice System. Unfortunately, there is still a high degree of stigma associated with $\mathrm{MH}{ }^{1}$

In August 2010, the Member of Parliament and former Royal Navy Doctor Andrew Murrison wrote a report entitled: 'Fighting Fit: A Mental Health Plan for Servicemen and Veterans'. ${ }^{1}$ The aim of this report was to address veterans' needs and provide extra MH support. In this report, Murrison explains that despite the $\mathrm{MH}$ services that are currently in place, service personnel with mental illnesses often do not seek help. The stigma associated with $\mathrm{MH}$, especially in the forces, is still prevalent and this has a part to play in the reasons why $\mathrm{MH}$ may not be diagnosed during active service. The report recognises that stigma is an issue within the military as a population that sees itself as mentally and physically robust. Additionally, it acknowledges the need to pass personnel back to civilian life in the best possible health. ${ }^{1}$ Significantly, the report also recommends that servicemen who are identified as requiring a specialist opinion during active service should be able to obtain it and any follow-on treatment from a military Department of Community Mental Health (DCMH) for a 6-month period once they have left the services. ${ }^{1}$

The report 'Fighting Fit' provides a number of recommendations all of which have the aim of improving $\mathrm{MH}$ services. Of the four principal recommendations, the 'Incorporation of a structured mental health system enquiry into existing 
medical examinations performed whilst serving' has led to this pilot study being conducted. The study aimed to assess the practicality of including an enhanced mental health assessment (EMHA) during routine and discharge medicals of serving personnel in order to detect MH Problems. An effective EMHA could facilitate earlier diagnosis and management and thus better $\mathrm{MH}$ outcomes for serving and ex-serving personnel.

\section{METHODS}

An EMHA questionnaire (Figure 1) was developed to highlight common $\mathrm{MH}$ problems such as depression, anxiety, alcohol misuse and post-traumatic stress disorder (PTSD) in keeping with the recommendations of the Murrison Report. ${ }^{1}$ The questions were taken from validated screening tools (Primary Care PTSD screen), ${ }^{5}$ Alcohol Use Disorder Identification Test (AUDIT-C), ${ }^{6}$ Generalised Anxiety Disorder assessment ${ }^{7}$ and Patient Health Questionnaire. $^{8}$

The questionnaires were to be completed as part of all routine and discharge medicals in the four participating medical centres (The Baird Medical Centre, the medical centres at the Army Training Centre (ATC) Pirbright, RAF Cranwell and Her Majesty's Naval Base, Clyde (HMNB)) over a 3-month period

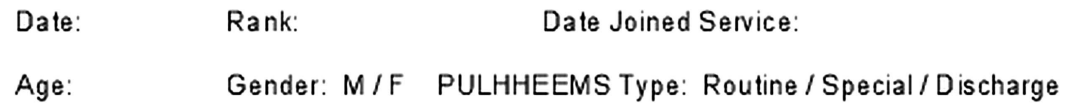

Depressed Mood

\begin{tabular}{|l|l|l|}
\hline Over the last month a persisting low mood & Yes & No \\
\hline Over the last month a loss of interest in pleasurable activities & Yes & No \\
\hline
\end{tabular}

Anxiety

Over the last month, a persisting feeling of being anxious, nervous

or on edge

\begin{tabular}{|l|l|l}
\hline Over the last month an inability to stop or control worrying thoughts & Yes & No
\end{tabular}

Post Traumatic Stress Symptoms

\begin{tabular}{|l|l|l}
\hline In the past month had nightmares about or had thoughts about an & Yes & No
\end{tabular}

unpleasant incident which were not wanted.

In the past month tried hard not to think about an unpleasant

incident or tried to avoid situations that remind them of itthem

In the past month has felt numb or detached from others, activities,

or their surroundings

In the past month has been constantly on guard, watchful or easily startled

Alcohol Use

How often does the person have

a drink containing alcohol

(Score)

\begin{tabular}{|l|} 
\\
\hline $\begin{array}{l}\text { How many units of alcohol do } \\
\text { you drink on a typical day when } \\
\text { drinking (Score) }\end{array}$ \\
\hline $\begin{array}{l}\text { How often had } 6 \text { or more units if } \\
\text { female, or } 8 \text { or more if male, on } \\
\text { a single occasion in the lastyear } \\
\text { (Score) }\end{array}$ \\
\hline
\end{tabular}

Sleep

In the last month had difficulty getting off to sleep or staying asleep

In the last month quality of sleep has interfered with day to day life

(you can prompt for daytime fatigue, ability to function at work/daily chores, concentration, memory, mood, etc.)

Anger / Irritability

In the last month has got angry with someone and yelled at them or threatened physical violence

Notes on results: Depression: $1 / 2$ Yes = possible Depression; $2 / 2$ Yes = probable Depression; Anxiety $1 / 2$ Yes $=$ possible anxiety disorder-investigate cause; PTSD: $2 / 4$ Yes = possible PT SD; $3 / 4$ Yes = Probable PTSD. Alcohol if scored ite ms add to more than 5, irwe stigate whether person drinking harmfully or dependently and assess person's concern about their drinking. Sle ep: If sleep is interfering with daily actirity, irwe stigate further for depression and anxiety or other reason for sleep disturbance. Anger: If positive inve stigate patient's concern about this. Outcome

No evidence of mental health problems

Some concerns, and patient offered advice and / or reassurance

Patient requires follow up and $\mathrm{PC}$ management

Patient requires $\mathrm{DCMH}$ referral

Patient declined DCMH referral and Civilian GP appraised of concerns

Patient is already referred or being managed by $\mathrm{DCMH}$

Figure 1 The Enhanced Mental Health Assessment tool. 
from May 2011 aiming to complete 100 questionnaires per centre. Prior to commencing the pilot study, each medical centre received a briefing and a copy of the EMHA questionnaire. The questionnaires were completed during consultations in the presence of the clinician. Patients were asked the questions as part of the consultation although they could refuse to answer the questions if they wished.

\section{Data handling}

Quantitative data were collated within a Microsoft Excel database for analysis. Qualitative data were central in identifying the feasibility of employing the EMHA. The aim was to gather information from the medical officers and practice managers about their participation in the pilot study via telephone interviews using a semi-structured questionnaire. A convenience sample of the personnel across the four medical centres was used and the qualitative results underwent trend analysis in order to identify any recurring themes.

\section{RESULTS}

Quantitative data

A total of 325 questionnaires from the planned 400 (81\%) were returned.

The modal average number of positive answers to the questions in the EMHA questionnaire was $0(n=273,84 \%)$. Overall, $2 \%(n=7)$ of patients had more than three positive answers. This included one patient who was already under psychiatric care scoring 11 positive answers, one patient scoring seven who received advice, one patient who scored six who required DCMH referral and four patients who scored four who were offered advice $(n=3)$ or showed no evidence of $\mathrm{MH}$ problems $(n=1)$. The overall outcomes are shown in Figure 2. One referral was made to a DCMH, and 8\% (26) of patients were categorised as 'some concern, and patient offered advice and/or reassurance'. The other outcome boxes represented only a handful of positive outcomes.

Data were collected on the alcohol consumption of British serving personnel, which identified 'higher risk drinking' in nearly two-thirds of responses. These results are discussed in more detail elsewhere. ${ }^{9}$

\section{Qualitative data}

Interviews were conducted with seven people (four doctors and three practice managers) on the basis of availability but all four medical centres were represented.

Prior to conducting the study, the initial feeling was that the EMHA was a hasty response to a government report and there was limited confidence that the study would provide any benefit in picking up $\mathrm{MH}$ problems during routine medicals. Interviewees felt that worries about stigma and possible effects on job progression could deter some patients from answering the questions honestly. There was also a considerable worry that implementing the questionnaire would require significant time and resources.

After completion of the pilot study, six interviewees felt that a $\mathrm{MH}$ assessment during discharge medicals was a useful screening tool. All felt that the questionnaire was quick and took up minimal extra time during consultations. However, the general feeling was that the EMHA did not reveal any problems that were not already evident from routine medicals. Some felt that a 3-month trial was insufficient and five people felt that the pilot study locations were not ideal such as phase I training establishments with a young, non-deployable population. They felt it would have been more beneficial to run the pilot study for a much longer period focusing on discharge medicals only. They all agreed that the idea behind it was good and that $\mathrm{MH}$ is an area that requires as much attention as the other aspects in a routine or discharge medical examination.

A number of recommendations arose from the telephone interviews. A tick box in the occupational health template on the Defence Medical Information Capability Programme (DMICP) reminding the doctor to address $\mathrm{MH}$ and alcohol consumption would be a desirable prompt. Another suggestion was that the military $\mathrm{MH}$ services should provide clear patient information leaflets to be handed out at discharge medicals for patients across all three services. These would detail all available $\mathrm{MH}$ services for veterans across the UK.

The alcohol section of the questionnaire was well received by all the interviewees. It was thought to have provided a very useful assessment tool and it was perceived that the format of the questions led to better and more honest answers from the patients. The consensus was that alcohol may be a significant problem among their patient populations and this tool may help to identify this problem more effectively.
Figure 2 The breakdown of number of patients per outcome from the enhanced mental health assessment. DCMH, Department of Community Mental Health; GP, general practitioner; $\mathrm{PC}$, primary care.

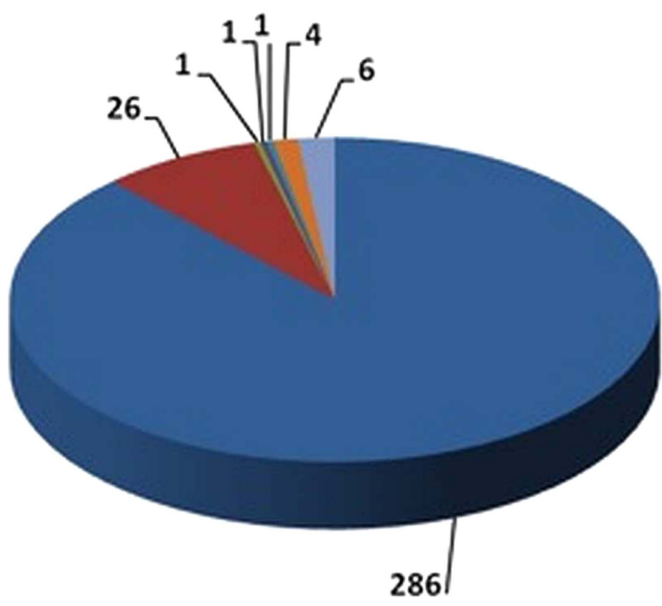

Noevidence of mental health problems

- Some concerns, and patient offered advice and/or reassurance

a Patient requires follow up and $\mathrm{PC}$ management

a Patient requires DCMH referral

a Patient declined DCMH referral and Civilian GP appraised of concerns

= Patient is already referred or being managed by $\mathrm{OCMH}$ 


\section{DISCUSSION}

$\mathrm{MH}$ in the UK Armed Forces

Serving in the Armed Forces can be a risk factor for the development of $\mathrm{MH}$ problems. ${ }^{10}$ Undoubtedly, military personnel do get exposed to stressful and traumatic situations, the scale of which is often beyond any comparable civilian experience and acute psychological distress can develop as a result. ${ }^{11}$ However, the most common $\mathrm{MH}$ disorders are anxiety and other more minor psychological conditions, and the prevalence of PTSD remains low. ${ }^{10}$

\section{MH screening}

Prevention of psychological morbidity

To date, no screening programme has been developed which could potentially detect $\mathrm{MH}$ problems prior to their manifestation. The American military implemented a selection programme in the Second World War with the aim of detecting individuals who could potentially develop $\mathrm{MH}$ problems. Initially, nearly two million men were removed from military service because they were highlighted as likely to break down; however, many were re-enlisted and the majority made satisfactory soldiers. ${ }^{12}$ There are many reasons why screening for $\mathrm{MH}$ susceptibility fails. In the American programme, one of the main reasons was lack of accuracy in the prediction methods. There is also a significant issue surrounding stigma and the perception of negative effects on career, which leads to a reluctance to admit to $\mathrm{MH}$ problems. ${ }^{12}$ Finally, in one study, screening produced a large number of false positives and false negatives, which highlighted that this type of screening programme would not provide a reliable method of identifying personnel at risk of developing $\mathrm{MH}$ problems. ${ }^{13}$ These issues are likely to have an impact on the findings of this pilot study.

\section{Deployment and screening for $\mathrm{MH}$ problems}

'Post Deployment Health Assessment' (PDHA) introduced by the US Army is a tool which screens soldiers upon return from deployment in order to promptly identify medical and $\mathrm{MH}$ problems. ${ }^{14}$ Studies have shown that the rates of reported deployment-related symptoms may increase with time after return from deployment. ${ }^{15-17}$ This led to an extension of the PDHA to include a re-evaluation at 3-6 months after return from a combat zone: 'Post Deployment Health Reassessment'. ${ }^{14}$ A significant proportion of the literature concentrates on screening using the above tools. However, there are also a number of studies which focus on pre-deployment screening, ${ }^{13} 1819$ or on screening personnel for specific $\mathrm{MH}$ disorders after they have presented to $\mathrm{MH}$ services. ${ }^{20}{ }^{21}$ Literature providing data on routine screening of military personnel regardless of deployment is scarce.

In this study, the assessment is conducted on personnel regardless of deployment history, so it may be difficult to compare the results with the available background literature.

\section{LIMITATIONS}

Our study does have significant limitations, which warrant discussion.

\section{Subjectivity}

Each medical officer implemented the questionnaire in a slightly different way. Some discussed each question with the patient while others placed the questionnaire in front of the patient for them to tick the boxes. In addition, the outcome box relies on the subjective judgement of the clinician. This may reduce the reliability and reproducibility of the results. One way in which this confounder was reduced was by the delivery of a brief to each medical centre prior to the pilot study where the questionnaire was explained and a set of criteria was given for when to tick each of the outcome boxes.

The subjective confounders may affect the reliability of the study results; conversely, it may be a true representation of how Defence $\mathrm{MH}$ assessments would be conducted in primary care should the EMHA be more widely implemented.

\section{Incomplete questionnaires}

Some questionnaires across each of the four medical centres had one or more questions or demographic details left blank leading to fewer questionnaires being fully useful for data analysis. The ideal solution to this would be to incorporate the template within DMICP.

\section{Outcome box not sufficiently comprehensive}

The questionnaire did not provide a separate outcome box for concerns regarding isolated alcohol problems. A medical officer may have deemed a particular patient to have some problems with alcohol consumption but not find any evidence of $\mathrm{MH}$ problems. Within the current questionnaire format, it would have been correct to tick 'no evidence of mental health problems' in the outcome box. However, another doctor may be tempted to tick 'some concerns and offered advice/reassurance'. A separate alcohol outcome box-Alcohol consumption of concern only'-may solve this problem. Other alcohol problems such as harmful use and dependence would go in the $\mathrm{MH}$ outcome boxes.

\section{Choice of pilot locations}

The Baird Medical Centre has a patient population, which consists predominantly of senior officers. Pirbright medical centre has a large patient population $90 \%$ of whom are recruits and the vast majority of medicals here were initial medical assessments in a population that has not been on deployment. At HMNB Clyde a large proportion of the population are young sailors who may undertake nuclear deterrent patrols but are unlikely to have deployed. RAF Cranwell is a training establishment with a high percentage of trainee pilots who may have reservations about the questionnaire due to fear of their training being affected.

\section{Deployment history}

There is evidence that deployment, especially in combat roles, has an association with $\mathrm{MH}$ problems: 'We also noted a significant association between deployment and probable post traumatic stress disorder in regular personnel who had a combat role during deployment ${ }^{3}{ }^{3}$ The literature provides potentially contradictory evidence regarding a link between number of deployments and $\mathrm{MH}$ problems. ${ }^{22}$ However, several reports have assessed duration of deployment in relation to health and have shown that psychological distress is positively associated with duration of deployment. ${ }^{22}{ }^{23}$ Potentially, a question regarding number and length of deployments in the EMHA would have been appropriate and relevant for the purposes of the questionnaire.

The literature and the qualitative data results from the study suggest that stigma remains a significant issue for the management of $\mathrm{MH}$ problems and we believe improved and increased education and awareness may encourage patients to seek help. 
Further research

This pilot study successfully demonstrated that the EMHA questionnaire was easy to administer, did not take up large amounts of resources or manpower and provided a potentially valuable assessment tool. A further study where the limitations identified are addressed may provide further data to validate and possibly implement the EMHA questionnaire. A repeat study should be for a minimum period of 6 months in a large Garrison setting, which incorporates deployable units and focuses solely on discharge medicals. The questionnaire itself should be available on DMICP and contain separate alcohol consumption outcome boxes.

\section{CONCLUSIONS}

The purpose of this pilot study was to assess the practicality of implementing the EMHA within routine and discharge medicals in UK Armed Forces. It demonstrated that this particular assessment tool would be easy to implement and would only require a minimal uplift in administrative resources, including time. The quantitative data have shown an average pick-up rate of $\mathrm{MH}$ problems. A further study using a more representative population could be used to assess the suitability of the EMHA as an assessment tool.

Acknowledgements We would like to thank Brigadier Cordell (DMS Head of Medical Strategy and Policy) for his support throughout this project. We would also like to thank Michael Rowe (Military Medical Librarian. Defence Medical Library Services) for his support with literature searches and background research, Amarjit Samra (Director of Research, JMC Medical Directorate) for invaluable advice on conducting research, and Anna Brown (Operations Manager, JMC Medical Directorate) for support with data analysis.

Contributors MA wrote the first draft with input from all authors. Each author has contributed to sections of the manuscript. Each author has edited the manuscript.

Competing interests None.

Patient consent Obtained.

Provenance and peer review Not commissioned; externally peer reviewed.

\section{REFERENCES}

1 Murrison A. Fighting fit. A mental health plan for servicemen and veterans. London: HM Government, 2010. https://www.gov.uk/government/publications/fighting-fit-amental-health-plan-for-servicemen-and-veterans-2

2 Greenberg N, Sharpley J. Advice to Military GPs-Mental Health Systems Enquiry at Routine PULHHEEMS and Discharge Medicals. 2010.

3 Fear NT, Jones M, Murphy D, et al. What are the consequences of deployment to Iraq and Afghanistan on the mental health of the UK Armed Forces? A cohort study. Lancet 2010:2;375:1783-97.
4 Iversen $\mathrm{AC}$, Greenberg N. Mental health of regular and reserve military veterans. Adv Psychiatr Treat 2009;15:100-6.

5 Prins A, Ouimette P, Kimerling R, et al. The primary care PTSD screen (PC-PTSD): development and operating characteristics. Int I Psychiatry Clin Pract 2004; 9:9-14.

6 Bush K, Kivlahan DR, McDonell MB, et al. The AUDIT alcohol consumption questions (AUDIT-C): an effective brief screening test for problem drinking. Ambulatory Care Quality Improvement Project (ACQUIP). Alcohol Use Disorders Identification Test. Arch Intern Med 1998;158:1789-95.

7 Spitzer RL, Kroenke K, Williams JBW, et al. A brief measure for assessing generalized anxiety disorder: the GAD-7. Arch Intern Med 2006;166:1092-7.

8 Gilbody S, Richards D, Brealey S, et al. Screening for depression in medical settings with the Patient Health Questionnaire (PHQ): a diagnostic meta analysis. J Gen Intern Med 2007;22:1596-602.

9 Aguirre M, Greenberg N, Sharpley J, et al. Alcohol consumption in the UK Armed Forces: are we drinking too much? J R Army Med Corps 2014;160:72-3.

10 Iversen AC, Staden LV, Hughes JH, et al. The prevalence of common mental disorders and PTSD in the UK military: using data from a clinical interview-based study. BMC Psychiatry 2009;9.

11 Section 5. How is psychological trauma managed in the Armed Forces? In: King's Centre for Military Health Research: a fifteen year report. London: King's College London 2010:31-2, 33.

12 Section 7. Mental health screening. In: King's Centre for Military Health Research: a fifteen year report. London: King's College London 2010:36-7.

13 Rona RJ, Hooper R, Jones $\mathrm{M}$, et al. Mental health screening in Armed Forces before the Iraq war and prevention of subsequent psychological morbidity: follow-up study. BMJ 2006;333:991.

14 Warner $\mathrm{CH}$, Appenzeller GN, Mullen K, et al. Soldier attitudes toward mental health screening and seeking care upon return from combat. Mil Med 2008:173:563-9.

15 Milliken CS, Auchterlonie JL, Hoge CW. Longitudinal assessment of mental health problems among active and reserve component soldiers returning from the Iraq war. JAMA 2007;298:2141-8.

16 Hoge CW, Castro CA, Messer SC, et al. Combat duty in Iraq and Afghanistan, mental health problems, and barriers to care. N Engl J Med 2004;351:13-22.

17 Bliese $\mathrm{D}$, Wright $\mathrm{M}$, Adler $\mathrm{B}$, et al. Timing of postcombat mental health assessments. Psychol Serv 2007;4:141-8.

18 Warner $\mathrm{H}$, Appenzeller $\mathrm{N}$, Parker $\mathrm{R}$, et al. Effectiveness of mental health screening and coordination of in-theatre care prior to deployment to Iraq: A cohort study. Am J Psych 2011;168:378-85.

19 Hyams KC. Mental health screening before troop deployment: is not supported by current evidence. BMJ 2006;333:979-80.

20 Gahm A, Lucenko A. Screening soldiers in outpatient care for mental health concerns. Mil Med 2008;173:17-24.

21 Felker B, Hawkins E, Dobie D, et al. Characteristics of deployed Operation Iraqi Freedom military personnel who seek mental health care. Mil Med 2008;173:155-8

22 Rona RJ, Fear NT, Hull L, et al. Mental health consequences of over-stretch in the UK Armed Forces: first phase of a cohort study. BMJ 2007;22;335:603.

23 Adler B, Huffman $H$, Bliese $D$, et al. The Impact of Deployment Length and Experience on the Well-Being of Male and Female Soldiers. J Occup Health Psychol 2005:10:121-37. 